\title{
Tri-Helical Gravure Roll Coating
}

\author{
R. W. Hewson, N. Kapur and P. H. Gaskell \\ Institute of Engineering Thermofluids, Surfaces and Interfaces, School of Mechanical \\ Engineering, University of Leeds, Leeds LS2 9JT, UK
}

\begin{abstract}
A mathematical model is presented and solved for the fluid flow within the coating bead of a tri-helical gravure roll coater, operating in reverse mode. A variety of rolls etched circumferentially with grooves of different cross sectional shape and aligned at non-zero angles of pitch are investigated. Predictions of fluid pick-out from the grooves are compared with complementary experimental data. Quantitative agreement between the two is found to be very good, showing a linear increase in pickout as a function of web-to-roll speed ratio and groove depths up to the point at which streaking, as observed experimentally, occurs and beyond which the model is no longer valid. In regions of parameter space for which there is no experimental data available for comparison purposes the model predicts that: (i) fluid pick-out decreases with increasing groove depth while the film thickness tends to increase; (ii) an increase in groove aspect ratio leads to a reduction in both pick-out and film thickness for a given land width and groove cross sectional shape.
\end{abstract}

Key words: Thin Films, Fluid Mechanics, Mathematical Modelling, Gravure Coating

\section{Introduction}

The use of a gravure roll for the deposition of continuous thin layers of fluid (typical viscosity, $\mu=1 \rightarrow 1500 \mathrm{mPas}$ ) with a wet thickness in the range 10 to $50 \mu \mathrm{m}$ on substrates moving at up to $10 \mathrm{~m} / \mathrm{s}$ is employed extensively in the coatings sector. The process, shown schematically in figure 1a, can be operated in either forward

Email addresses: r.w.hewson@leeds.ac.uk (R. W. Hewson), n.kapur@leeds.ac.uk (N. Kapur),p.h.gaskell@leeds.ac.uk (P. H. Gaskell). 
or reverse mode, the latter being the more common due to its preferential stability characteristics (Benkreira \& Cohu 1998).

[Fig. 1 here.]

The authors' recent paper, Hewson et al. (2006), provides a thorough review of the current state of the art; accordingly to avoid unnecessary repetition it is sufficient to state that for gravure rolls, consisting of laser engraved or mechanically knurled cells on an otherwise smooth roll surface, the film thicknesses produced depend primarily on two factors: (i) the doctoring process (Patel \& Benkreira 1991, Pranckh \& Coyle 1997); (ii) the fluid transfer between gravure cells and moving substrate (Pulkrabek \& Munter 1983, Kapur 2003, Kapur et al. 2001, Powell et al. 2000, Schwartz 2002). Consequently both the geometry of the gravure cells, the web and roll speeds, web path and tension and fluid properties can all influence the coat thickness. Gravure rolls can be sub-divided into two primary classes - cells that form a continuous channel from the upstream to downstream of the bead or discrete cells which are lengthscales smaller than the coating bead which can be regarded as pockets of fluid. A typical cell size is of the order of 10 to $100 \mu \mathrm{m}$ depending on the desired coat weight.

While satisfactory modelling of the discrete process has remained elusive compared to its smooth roll counterpart, kiss coating (Gaskell et al. 1998), the continuous groove form, namely tri-helical gravure roll coating, has proved more amenable to mathematical analysis, but until now only for the particular case of rectangular groove etched, at a zero angle of pitch, $\Phi$ (measured normal to the roll axis of rotation), circumferentially over the entire surface of the roll (Hewson et al. 2006). In practice grooves etched at a non-zero pitch angle are the norm, as are ones that are not strictly rectangular in cross section; two factors which represent a further considerable modelling challenge, the former more so than the latter.

The motivation and thrust of the present study is, therefore, the formulation of a predictive mathematical model for the more general case of tri-helical gravure roll coating which caters for rolls having a groove etched into them of arbitrary cross sectional shape, aligned at a non-zero angle of pitch. This important extension to the authors' earlier model (Hewson et al. 2006) accounts for the cross flow perpendicular to the groove direction as well as embodying the case of non-rectangular groove cross-sections. The model is described in Section 2; this is followed by a brief outline of the complementary experiments that were carried out for validation 
purposes, Section 3. A comprehensive set of results is provided in Section 4, with particular attention given to predictions of the volume of fluid transferred to the web for differing groove geometries when operated in the stable coating regime. Conclusions are drawn in Section 5.

\section{Mathematical Model and Method of Solution}

Consider, as is illustrated schematically in Figure 1, the case of an infinitely long tri-helical gravure roll coater operating in reverse mode. The liquid forming the two-dimensional cross-sectional coating bead is assumed to be incompressible with constant viscosity $\mu$, density $\rho$ and surface tension $\sigma$.

[Fig. 2 here.]

The following scalings are used throughout the subsequent analysis:

$$
\left[x, y, b, c, r_{\mathrm{roll}}\right]=\frac{\left[\underline{X}, B, C, D, R_{\mathrm{roll}}\right]}{A} \quad[u, v, S]=\frac{\left[\underline{U}, U_{\mathrm{web}}\right]}{U_{\mathrm{roll}}} \quad p=\frac{P A}{\mu U_{\mathrm{roll}}},
$$

where: $\underline{X}=(X, Y)$ represent the chosen Cartesian frame of reference; $\underline{U}=(U, V)$ are the corresponding coordinate specific velocity components; $A, B, C, D$ and $R_{\text {roll }}$ are the dimensions representing groove width, land width, groove depth (see figure 2c), web-to-roll gap and roll radius, respectively; $U_{\text {web }}$ and $U_{\text {roll }}$ are the web and peripheral roll speed, respectively; $S$ is the web-to-roll speed ratio and $P$ the pressure. As a consequence of the scalings adopted the non-dimensional groove depth, $c$, is also the groove aspect ratio.

\subsection{Groove Flux}

The total flux, $q$, per groove is found by determining separate longitudinal, $q_{x^{\prime}}$, and transverse, $q_{y^{\prime}}$, components and summing them together - see figure $2 \mathrm{a}$.

$$
q=q_{x^{\prime}}+q_{y^{\prime}}
$$




\subsubsection{Derivation of the longitudinal flux, $q_{x^{\prime}}$}

The longitudinal flux is bounded by the web which is moving with speed $u^{\prime}=$ $S \cos \Phi$ and the sides of the groove having speed $u^{\prime}=\cos \Phi$. Neglecting inertial terms, the $x^{\prime}$ momentum Navier-Stokes equation simplifies to that of a two dimensional Poisson equation (Hewson et al. 2006) of the form:

$$
\frac{\partial p}{\partial x^{\prime}}=\frac{\partial^{2} u^{\prime}}{\partial y^{\prime 2}}+\frac{\partial^{2} u^{\prime}}{\partial z^{2}}
$$

Unlike the case of grooves with a rectangular cross-section, an analytical solution to equation (3) for grooves of arbitrary cross sectional shape does not exist. Accordingly, a numerical solution has to be found. For this purpose a Galerkin finite element finite element (FE) formulation was employed, with linear interpolation between nodes, resulting in the following equation for one such element:

$$
\iint_{\Omega}\left[\frac{\partial N_{i}}{\partial y} \frac{\partial N_{j}}{\partial y}+\frac{\partial N_{i}}{\partial z} \frac{\partial N_{j}}{\partial z}\right] d y d z u_{i}=\iint_{\Omega} \frac{d p}{d x} N_{i} d \Omega .
$$

where $N$ are linear interpolation functions; $\Omega$ is the element area and $d \Omega$ its boundary; the corresponding solution domain, exploiting the symmetry of the problem, and associated boundary conditions are shown in figure $2 \mathrm{~b}$. The total number of elements required to produce grid independent solutions in the $x^{\prime}$ direction was found to depend on the size of the web-to-roll gap (as defined in figure 1b), with use of the order of 100 elements proving sufficient in all case. The $u^{\prime}$ velocity obtained is then numerically integrated across the groove to give the volumetric flow rate $q_{x^{\prime}}$.

\subsubsection{Derivation of the transverse flux, $q_{y^{\prime}}$}

Determination of the transverse flux necessitates a series of further simplifications, enabling it to be found as the sum of two parts, namely:

(1) The flux contained within the grooves, $q_{1 y^{\prime}}$, is assumed to be transported by virtue of the liquid being in contact with the roll and the product of (i) the equivalent film thickness (the average depth of fluid on the roll surface), (ii) the length the flux passes though per groove $((1+b) \tan \Phi)$ and (iii) the speed 
of the roll in the $y^{\prime}$ direction, $\sin \Phi$. In which case:

$q_{1 y^{\prime}}=\underbrace{k}_{\text {average depth of fluid }} \times \underbrace{(1+b) \tan \Phi}_{\text {groove dimension projected in the } y^{\prime} \text { direction }} \times \underbrace{\sin \Phi}_{\text {roll speed in the } y^{\prime} \text { direction }}$

(2) In the gap between the web and the roll surface the flow is assumed to be a combination of Poiseuille and Couette like flow:

$$
\frac{\partial^{2} v^{\prime}}{\partial z^{2}}=\frac{\partial p}{\partial y^{\prime}} \equiv \frac{\partial p}{\partial x} \sin \Phi
$$

When integrated twice with respect to $z$ equation (6) gives:

$$
v^{\prime}=\frac{z^{2}}{2} \frac{d p}{d x} \sin \Phi+z c_{1}+c_{2}
$$

where $c_{1}$ and $c_{2}$ are arbitrary constants to be determined. Due to the varying geometry in the $y^{\prime}$ direction, the average depth of fluid, $k$, is used and at this location - see figure $2 \mathrm{c}$ - with a speed which is assumed to be that of the roll. The boundary conditions required to obtain $c_{1}$ and $c_{2}$ are the web and roll surface speeds, given by:

$$
v^{\prime}=\sin \Phi \quad \text { at } \quad z=0 \quad \text { and } \quad v^{\prime}=S \sin \Phi \quad \text { at } \quad z=d+k
$$

such that,

$$
c_{1}=\frac{2 S-2-\sin \Phi(d+k)^{2} \frac{d p}{d x}}{2 d+2 k} \quad \text { and } \quad c_{2}=\sin \Phi .
$$

Hence from equation (7)

$$
v^{\prime}=\frac{\left(z^{2} \frac{d p}{d x}(d+k)+\left(-(d+k)^{2} \frac{d p}{d x}+2 S-2\right) z+2 d+2 k\right) \sin (\Phi)}{2 d+2 k} .
$$

The flux per unit width in the gap between the web and the roll surface, $q_{2 y^{\prime}}$, is obtained by integrating equation (10), the limits of integration being the land and web surfaces. In effect this gives the flow between the web and land a certain degree of slip at the roll surface. The physical manifestation of this is 
that it accounts for the grooves located beneath the roll lands. Accordingly:

$$
\begin{aligned}
q_{2 y^{\prime}}= & \frac{\sin (\Phi) d(d+2 k)(1+b) \tan (\Phi)}{2 d+2 k} S \\
& -\frac{\sin (\Phi) d^{2}(d+3 k)(d+k)(1+b) \tan (\Phi)}{12 d+12 k} \frac{d p}{d x} \\
& +\frac{\sin (\Phi)(1+b) \tan (\Phi)\left(d^{2}+2 d k+2 k^{2}\right)}{2 d+2 k} .
\end{aligned}
$$

Hence,

$$
q_{y^{\prime}}=q_{1 y^{\prime}}+q_{2 y^{\prime}}
$$

\subsection{Pressure Gradient}

Equation (2) is used to find the associated pressure distribution throughout the coating bead. From conservation of mass considerations, the pressure gradient is an unknown variable and dependent on roll geometry, operating conditions and flux but is readily determined due to the linear relationship between flux and pressure gradient (as seen by inspecting equations (3) and (11)).

The value of the pressure at the upstream and downstream menisci, together with their respective locations is required to make the pressure equation tractable. Accordingly, a meniscus model is required that relates the operating conditions to the film thickness and local radius of curvature, which together with surface tension determines the pressure discontinuity across the meniscus. This is achieved using the cavitation model due to Coyne \& Elrod (1970), Coyne (1967) for the withdrawal of a plate from a flooded gap - see Hewson et al. (2006).

\subsection{Meniscus Models}

Descriptions of the downstream and upstream menisci are based on the model of Coyne (1967), Coyne \& Elrod (1970) which enables the deposited film to gap ratio and free surface curvature to be calculated as a function of capillary number.

On the downstream side the grooves enter the coating bead full, and the twodimensional meniscus that is formed there runs the length of the roll. This has been verified experimentally by Hewson et al. (2006) for the case when there is web-toroll contact, as the average velocity of the fluid in the groove is lower than the roll 
surface speed. At the upstream side, the partially full grooves exiting the coating bead result in the meniscus there dipping into the grooves and forming a complex three-dimensional shape. A simplified geometry for the upstream meniscus is therefore required; the one adopted is based on an equivalent rectangular geometry to that of the actual groove cross-sectional shape under consideration, see figure 3 . For rectangular grooves the actual groove geometry itself is used $\left(a^{\prime}=1, b^{\prime}=b\right)$. For triangular grooves, on the other hand, the line width, groove depth and groove cross sectional areas are conserved resulting in the following dimesions, $a^{\prime}=0.5$ and $b^{\prime}=b+0.5$.

[Fig. 3 here.]

The downstream meniscus model employed is similar to that used for the case of zero pitch angle, see Hewson et al. (2006), with the line width (the projection of the groove length in the $y, z$ plane) corrected for the groove pitch angle, $\left(a^{\prime}+b^{\prime}\right) \cos \Phi$. The film thickness $\left(h_{d s m}\right)$ coated onto the web is related to flux, speed ratio and line width $\left(\left(a^{\prime}+b^{\prime}\right) \cos \Phi\right)$ by:

$$
h_{d s m}=\frac{q}{\left(a^{\prime}+b^{\prime}\right) S \cos \Phi} .
$$

Using a standard parabolic approximation for the local roll surface shape (Gatcombe 1945) enables the location of downstream meniscus, $d$, to be determined:

$$
d=\frac{x^{2}}{2 r_{\text {roll }}} \quad(x<0) \quad \text { and } \quad d=\frac{\left(x-\beta r_{\text {roll }}\right)^{2}}{2 r_{\text {roll }}} \quad(x>\beta),
$$

where $\beta$ is the wrap angle and $r_{\text {roll }}$ is the roll radius. Following this, the local radius of curvature is then used to obtain the pressure there.

The upstream meniscus model is akin that used by Hewson et al. (2006) to calculate the pressure and location for the zero ptched groove case. The method is based on a representative two dimensional meniscus relating the geometry to the free surface. The fluid film left on the roll surface is shown in figure 3 , with $h_{1}, h_{2}$ and $h_{3}$ given by:

$$
h_{1}=\frac{c+d}{h_{\theta}} \quad h_{2}=\frac{\cos \Phi}{2 a^{\prime} h_{90}} \quad h_{3}=\frac{d}{h_{\theta}},
$$

where $h_{\theta}$ is the gap to film thickness ratios at a contact angle of $\theta$ (the dynamic contact angle), where the meniscus meets the web. Simplifying the problem further by neglecting the curvature of the meniscus in the corners of the partially filled grooves (the inside and outside corners will cancel each other out to some degree) 
the flux on the roll can be written as:

$$
q_{\mathrm{roll}}=\frac{a^{\prime} h_{1}}{\cos \Phi}+h_{2}\left(c-h_{1}+h_{3}\right)+\frac{h_{3} b^{\prime}}{\cos \Phi},
$$

which after substitution of equations (15) can be rearranged to solve for the roll-toweb gap, $d$, which in turn is used to determine the location of the meniscus from the parabolic approximation of the roll surface (equation (14)) for $x>\beta r$.

When curved in two dimensions the meniscus pressure is calculated thus:

$$
p_{u s m}=\frac{\kappa_{y}+\kappa_{z}}{C a}
$$

where $\kappa_{y}$ and $\kappa_{z}$ are the meniscus curvatures in the $y$ and $z$-directions. $\kappa_{y}$ is based on the two different radii of curvature, that between the land and web $\left(\kappa_{y 2}\right)$ and the other between the groove base and web $\left(\kappa_{y 1}\right)$ :

$$
\kappa_{y}=\frac{\left(a^{\prime} \kappa_{y 1}+b \kappa_{y 2}\right)}{a^{\prime}+b^{\prime}}
$$

$\kappa_{z}$ is simply based on the width of the groove. This leads to the following equation for the upstream meniscus pressure:

$$
p_{u s m}=\frac{1}{C a}\left(\kappa_{z}+\frac{a^{\prime} \kappa_{y 1}+b^{\prime} \kappa_{y 2}}{a^{\prime}+b}\right) .
$$

\subsection{Calculation Procedure}

A method of searching is adopted in order to calculate the total flux and corresponding pressure distribution. The procedure is essentially that described in Hewson et al. (2006), although here the flow field is calculated using the finite element method. The flux is varied incrementally in order to obtain a unique pressure distribution of the flow, equation (3), which is consistent with the pressures at the two bounding menisci.

\section{Experimental Method}

The experimental data collected with which to compare with predition was obtained using existing tried and tested apparatus and procedures (Hewson et al. 2006). As 
in their earlier experiments, use was made of acrylic sleeves with grooves cut radially into the surface and mounted on a steel core with adjustable grub screws at either end to ensure concentricity (runout of less than $0.05 \mathrm{~mm}$ was achieved) grooves of both rectangular and triangular cross section were considered, the dimensions (taken in the plane perpendicular to the groove direction) of which are given in figure $2 \mathrm{c}$ - the corresponding dimensional values are provided in Table 1. The incoming web was passed under an idle roll mounted on an eccentric journal allowing the wrap angle to be varied between $0^{\circ}$ and $5^{\circ}$.

[Table 1 here.]

After start up and once the process had stabilised, the fluid transferred to the web was removed with a flexible rubber blade held firmly against the web by means of a backing roll. The fluid collected was then used to determine the coated film thickness from which the pickout, $\Psi$, could be calculated using the following equations:

$$
\begin{aligned}
& \Psi_{T}=\frac{\text { Flux leaving on web }}{\text { Flux entering within grooves }}=\frac{2 U_{\text {web }} H(A+B)}{U_{\text {roll }} A C} ; \\
& \Psi_{R}=\frac{\text { Flux leaving on web }}{\text { Flux entering within grooves }}=\frac{U_{\text {web }} H(A+B)}{U_{\text {roll }} A C} ;
\end{aligned}
$$

for triangular (T) and rectangular (R) grooved rolls, respectively. $H$ is the coated film thickness. Values of $A, B$ and $C$ in each case are provided in Table 1.

\section{Results}

Results from a systematic investigation of tri-helical gravure roll coating are presented with comparison between experimental data where it exists and prediction made. Arguably the result of most practical importance when operating in a stable coating regime is the volume of fluid transferred from a tri-helical gravure roll to a web which, as described above, can be expressed as either the film thickness deposited on the web or as a fractional pickout via equation (20) or (21). 


\subsection{Groves at Zero Angle of Pitch Revisited}

For the case of rectangular grooves comparison is made with experimental data and the predictions obtained by Hewson et al. (2006) using their analytical model. The comparison is shown in figure 4, were the level of agreement between all three sets of data is seen to be particularly good, with the results predicted by the present model lying closer to and being in better agreement with experiment.

[Fig. 4 here.]

The model is now used to explore pickout as a function of speed ratio for the case of two different triangular groove geometries at a zero angle of pitch. These are shown in Figures 5a and 5b. As before, agreement between theory and experiment is seen to be good with pickout being slightly over predicted, up to the point where maximum pickout occurs. As in the case of rectangular grooves, pickout increase with speed ratio up to a value of $S$ of approximately 1.2, after which corresponding experiments reveal that streaking occurs (the exact value depends on the specific operating conditions). The onset of the streaking instability arises when the upstream meniscus moves entirely within the groove and passes downstream to merge with the meniscus there causing a dry patch on the web (Hewson et al. 2006). This feature consequently provides an upper bound beyond which the model is no longer valid; this is due to the limitations of the meniscus model employed which assumes a continuous meniscus. After the onset of streaking the transient nature of the upstream meniscus location suggests that there is an unstable coupling between the pressure gradients in the coating bead and the location of the upstream meniscus - leading to the latter moving backwards and forwards along the grooves, forming streaks.

[Fig. 5 here.]

\subsection{Grooves with Non-zero Angle of Pitch}

Results for the case of rolls with grooves at non-zero angles of pitch are given in figure 6. In all cases an increase in speed ratio leads to a corresponding increase in pickout, up to some maximum, before streaking occurs. Prior to this point being reached, the agreement between predicted and measured values is extremely good for pitch angles of $30^{\circ}$ and $45^{\circ}$; beyond $S=1.5$ the continued rise in pickout 
observed experimentally fails to be captured by the model, which is likely due to the groove behaving more like a series of discrete cells as the upper limit of pitch angle, $90^{\circ}$, is approached. Under such conditions the grooves become essentially aligned with the roll, forming longitudinal two-dimensional cells, i.e. the upstream and downstream menisci are no longer joined by a continuous groove. Also, inherent in the model's formulation is the assumption of a non-deformable web which leads to the prediction of a zero flux in such circumstances - which is clearly not the case in practice, since discrete cell gravure coating is a viable process; this decrease in pickout with increasing groove angle is illustrated in figure 7.

Agreement between prediction and measurement for grooves pitched at $60^{\circ}$ is less satisfactory (figures 6e and 6f), being reasonable only for low values of $S$. The level of agreement between the experimental and model results for roll T5 (figure 6d) is fairly poor - the most likely reason for this is that the land width is significantly larger than the groove making it much more susceptible to experimental error due to the low volume of fluid entering the coating bead within the grooves.

[Fig. 6 here.]

[Fig. 7 here.]

[Fig. 8 here.]

Figure 8 shows the predicted pressure distribution through the coating bead as the groove pitch angle is varied for the case of rectangular grooves with capillary number $C a=0.068$ and speed ratio $S=1$. At low pitch angles the predicted pressures are entirely sub-ambient. In terms of its effect on the path of flexible substrates as encountered in practice, such a pressure gradient would effectively "suck" the web down towards the roll surface with the interplay between the lands and the tension in the web preventing it from being pulled into the grooves. For pitch angles greater than $60^{\circ}$ the model predicts a large positive pressure over a large section of the coating bead. In practice, such a pressure distribution would force the web away from the roll; once again, as web flexibility is not embodied within the model this is a further reason for its failure to capture the dynamics of the process at high angles of pitch. 


\subsection{Prediction of the Effect of Groove Aspect Ratio}

A parametric study of the effect of groove aspect ratio on pickout and film thickness was carried out. In doing so the groove cross-sectional area was kept constant for a given change in the groove aspect ratio. Since dimensions are scaled with groove width (resulting in the groove aspect ratio being the same as the non-dimensional groove depth), see equations (1), then as the aspect ratio is varied other affected parameters have to be scaled accordingly in order to maintain the line width $(A+B)$ and fluid volume entering the coating bead.

[Table 2 here.]

The dimensions in Table 2 were used to obtain the non-dimensional variables $\beta, l$, $r_{\text {roll }}$ and $c$ from the following equations, with $A$ varied from 0.4 to 0.9 :

$$
\begin{gathered}
c=\frac{0.5}{A^{2}}, \\
b=\frac{1}{A}-1, \\
r_{\text {roll }}=\frac{50}{A} .
\end{gathered}
$$

Figures 9a and 9b show typical predicted results for pickout (left-hand vertical axis) as a function of groove aspect ratio for groove pitch angles of $0^{\circ}$ and $45^{\circ}$. They show that as the groove aspect ratio increases (i.e. the grooves get narrower and deeper) then, as expected, pickout falls. Since the fluid volume entering the coating bead is constant, the film thickness is directly proportional to pickout and is given on the right-hand vertical axis.

[Fig. 9 here.]

As the model is no longer valid once the onset of streaking occurs care must be taken when examining results; in practice streaking may occur at a lower speed ratio for shallow groove depths - it is for this reason that results for speed ratios above $S=1$ are not shown. 


\subsection{Effect of Groove Depth}

The effect of groove depth is now examined. Increasing the groove depth without reducing the groove width means that the volume of fluid entering the coating bead increases; figures $10 \mathrm{a}$ and $10 \mathrm{~b}$ show that as the groove depth increases the pickout decreases. This is in line with the results of section 4.3 , with the motion of the web having a diminishing influence on the fluid at the base of a groove as the depth increases. This effect is observed for grooves having a zero pitch angle and, the more industrially representative, grooves pitched at $45^{\circ}$.

[Fig. 10 here.]

The effect of groove depth on the film thickness deposited onto the web is influenced not only by the pickout from the grooves but also by the volume of fluid entering the coating bead. Although pickout decreases as the groove depth increases, a greater volume of fluid entering the coating bead leads to an increase in the deposited film thickness (as shown in figures 11a and 11b) followed by a fall in film thickness as the groove depth increases further. This decrease in film thickness can be attributed to the pressure driven flow within the coating bead.

[Fig. 11 here.]

For larger groove depths the pressure distribution between the two bounding menisci is such that there is an additional pumping of fluid from the downstream to the upstream side, resulting in a decrease in the film thickness. This is illustrated in figure 12 which shows a typical velocity profile along the groove centerline for four different groove depths. The "bulge" in the profile as shown in figures $12 \mathrm{c}$ and $12 \mathrm{~d}$ illustrates the increase in velocity, resulting in a tube of fluid moving within the groove with a velocity greater than that of the roll, thereby reducing the film thickness.

[Fig. 12 here.]

As shown in section 4.2, as the pitch angle increases the relatively high pressures at the downstream meniscus decrease and the relatively low pressures at the upstream meniscus increase, reducing the pressure gradients from the downstream to the upstream side, thereby giving less pressure driven pumping. 


\subsection{Effect of Groove Shape}

\subsubsection{Rectangular, Trapezoidal and Triangular Cross-section}

The effect of systematically changing the groove shape from that of rectangular, through trapezoidal to triangular, as shown in figure 13a, is examined.

[Fig. 13 here.]

The width of the groove opening $(A)$ is kept constant by varying the groove depth as the width of the groove base is changed. For the geometries explored, the predictions show that there is a clear decrease in pickout and film thickness as the grooves become more triangular in shape and thus deepened- see figures $14 \mathrm{a}$ and $14 \mathrm{~b}$ for grooves pitched at $0^{\circ}$ and $45^{\circ}$, respectively. The increased pickout for rectangular grooved rolls is due to the greater motion imparted to the fluid by the web due to the shallower groove geometry.

[Fig. 14 here.]

A further interesting result is the predicted variation of film thickness with groove geometry as shown in figures $15 \mathrm{a}$ and $15 \mathrm{~b}$. It can be clearly seen that the film thickness for $S=0.5$ and $S=1$ cross, highlighting the complex relationship between film thickness profiles and speed ratio, and suggesting that it may be possible to design grooved rolls to give a film thickness that is relatively insensitive to the speed ratio (or conversely, one that maximises the sensitivity allowing variability to be "designed in" to the process).

[Fig. 15 here.]

\subsubsection{Triangular, Rectangular and Semi-Circular Cross-section}

The effect of three different groove geometries, as shown in figure 13b, is examined. Each of these roll geometries have the same cross-sectional area and width of opening. The corresponding pickout characteristics are shown in figures 16a and 16b. It can be seen that the semi-circular and rectangular grooves predict a higher pickout than the deeper triangular ones. This is consistent with the case studied in section 4.5.1 and is likely to be due to the moving web being further away from the fluid at the apex of the triangular grooves, thereby imparting less motion to the fluid 
in the web direction.

[Fig. 16 here.]

For grooves of zero pitch, those of a semi-circular cross-sectional profile predict a slightly greater pickout than those of a rectangular cross section; however at a pitch of $45^{\circ}$ the pickout achieved with rectangular grooves is greater. From this it is possible to deduce that the web imparts a greater motion to the fluid in the case of semi-circular grooves; however, the pumping of fluid due to the induced pressure gradient is more important for rectangular grooves. Since the pressure gradient for rolls with grooves of greater pitch is more significant, the difference between grooves of rectangular and semi-circular cross-section (and to some extent triangular grooved rolls) is reduced.

\section{Conclusion}

A model of the tri-helical gravure roll coating process for the general case of rolls with grooves of fixed arbitrary cross section and aligned at non-zero angles of pitch is presented and shown to predict film thickness and hence pickout over a wide range of parameter space. In particular:

- Where complimentary experimental data is available, there is found to be good quantitative agreement with prediction.

- It is shown that for groves having a zero angle of pitch that as the web-to-roll speed ratio increases up to a value of approximately $|S| \approx 1.2$ the corresponding pickout increases approximately linearly. After this point streaking is observed experimentally and beyond which the model breaks down.

- As the groove pitch angle is increased there is a decrease in pickout up to the point where the problem becomes more like that of discrete cell gravure roll coating and elastohydrodynamic effects become important due to the increase in pressures within the coating bead.

- The effects of groove shape reveals (i) that pickout decreases with increasing groove depth while the film thickness tends to increase; (ii) that an increase in groove aspect ratio leads to a reduction in the pickout and film thickness for a given line width and groove cross sectional shape. 
The ability of the model to provide film thickness and pickout predictions for a variety of factors influencing groove geometry make it a useful addition for designing new tri-helical gravure rolls as well as for gaining an understanding of how operating parameters effect the overall coating process.

\section{Acknowledgments}

This work was funded by the Engineering and Physical Sciences Research Council UK, grant number GR/R70064/01, with additional support from Dupont Teijin Films UK Ltd.

\section{References}

Benkreira, H. \& Cohu, O. (1998), 'Direct forward gravure coating on unsupported web', Chemical Engineering Science 53(6), 1223-1231.

Coyne, J. (1967), Conditions for the rupture of a lubricating film, Eng. sci. d. dissertation, Columbia.

Coyne, J. C. \& Elrod, H. G. (1970), 'Conditions for the rupture of a lubricating film. Part 1: Theoretical model.', Journal of Lubrication Technology 92, 451.

Gaskell, P. H., Rees, S. R., Savage, M. D. \& Storey, S. P. (1998), 'A mathematical model of roll-to-web kiss coating', Chemical Engineering Research and Design 76(A1), 29-37.

Gatcombe, E. (1945), 'Lubrication characteristics of involute spur gears', Transactions of the ASME 67, 177.

Hewson, R. W., Kapur, N. \& Gaskell, P. H. (2006), 'A theoretical and experimental investigation of tri-helical gravure roll coating', Chemical Engineering Science 61(16), 5487-5499.

Kapur, N. (2003), 'A parametric study of direct gravure coating', Chemical Engineering Science 58(13), 2875-2882.

Kapur, N., Gaskell, P. H. \& Bates, A. (2001), 'A parametric study of offset gravure coating', Chemical Engineering Research and Design 79(A1), 41-50.

Patel, R. \& Benkreira, H. (1991), 'Gravure roll coating of Newtonian liquids', Chemical Engineering Science 46(3), 751-756.

Powell, C. A., Savage, M. D. \& Gaskell, P. H. (2000), 'Modelling the meniscus 
evacuation problem in direct gravure coating', Chemical Engineering Research and Design 78(A1), 61-67.

Pranckh, F. R. \& Coyle, D. J. (1997), Liquid film coating scientific principles and their technological implications, Chapter 12b, Elastohydrodynamic Coating Systems, Chapman \& Hall, London.

Pulkrabek, W. W. \& Munter, J. D. (1983), 'Knurl roll design for stable rotogravure coating', Chemical Engineering Science 38(8), 1309-1314.

Schwartz, L. W. (2002), 'Numerical modeling of liquid withdrawal from gravure cavities in coating operations; the effect of cell pattern', Journal of Engineering Mathematics 42(3-4), 243-253. 


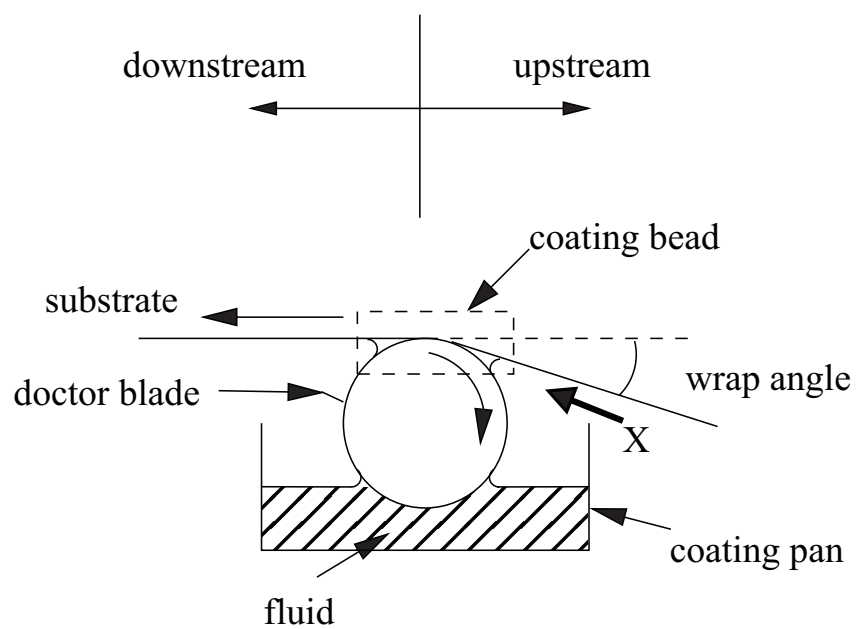

(a)

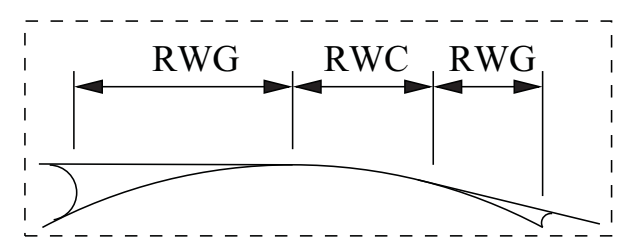

(b)

Fig. 1. (Cross sectional schematic of a reverse gravure roll coater operating in reverse mode showing an exploded view of the two-dimensional coating bead indicating the associated roll-to-web contact (RWC) region and the roll-to-web gap (RWG) to either side. 


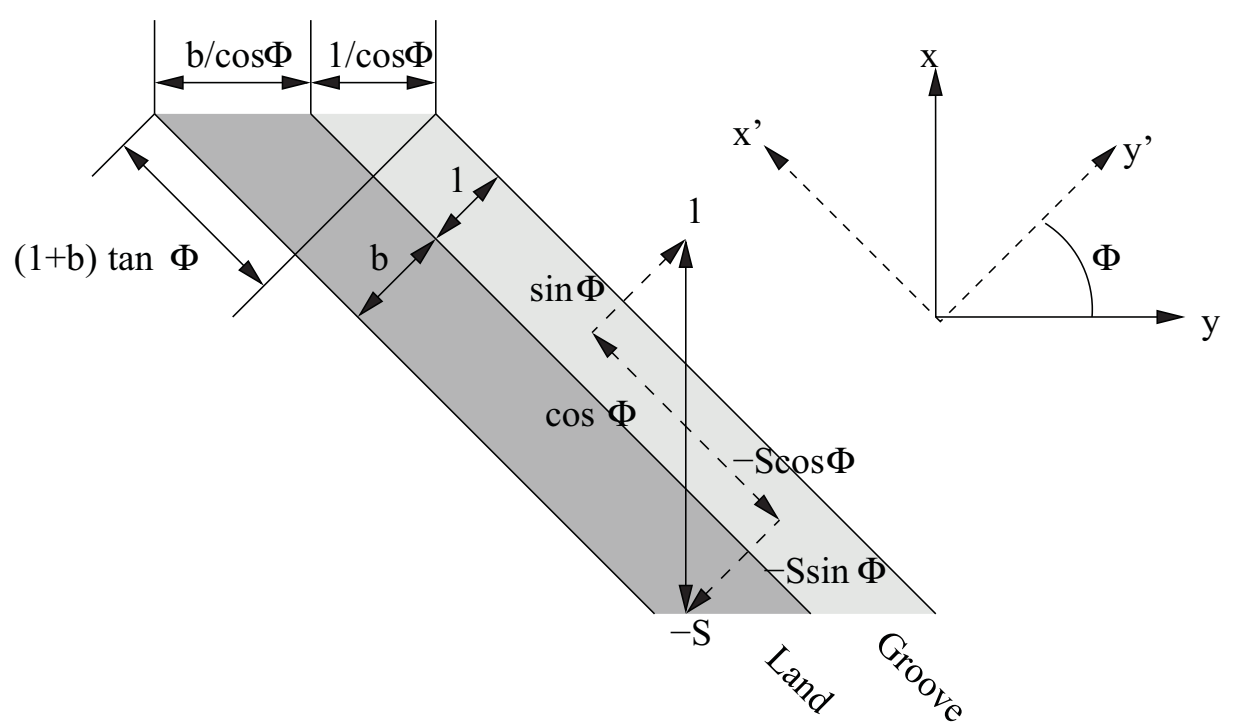

(a)

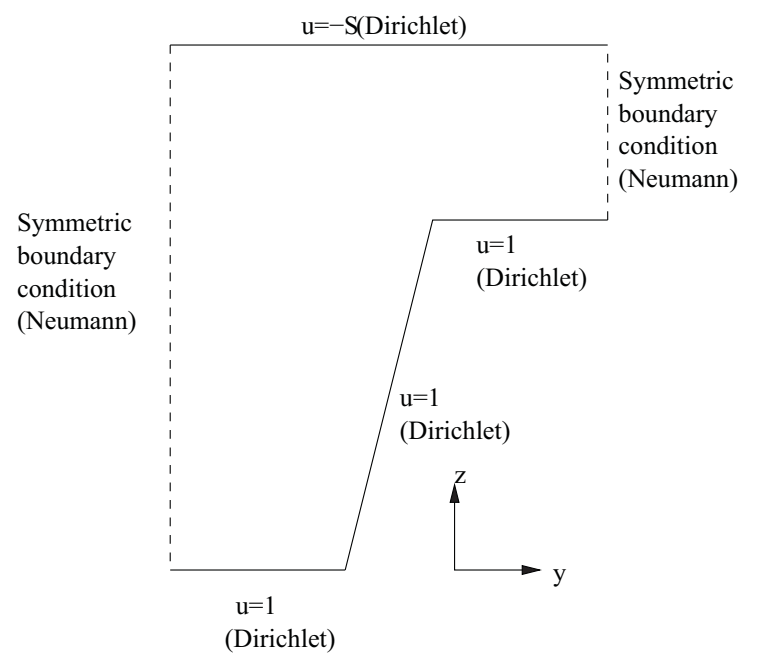

(b)

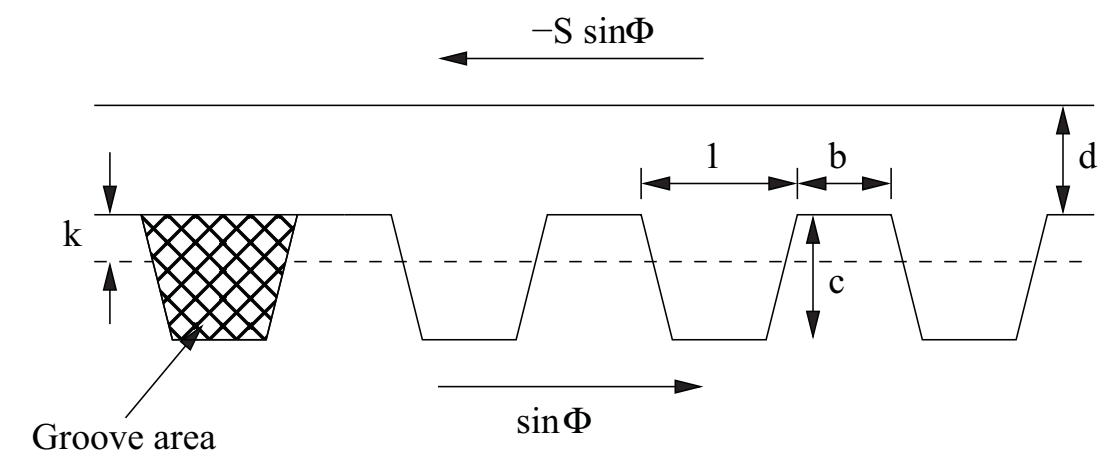

(c)

Fig. 2. (a) Resolution of velocities, (b) symmetric solution domain boundary conditions (c) cross-flow geometryshowing characteristic tri-helical groove dimensions - groove width, $A$, land width , $B$, groove depth, $C$, web-to-roll gap, $D /$ 


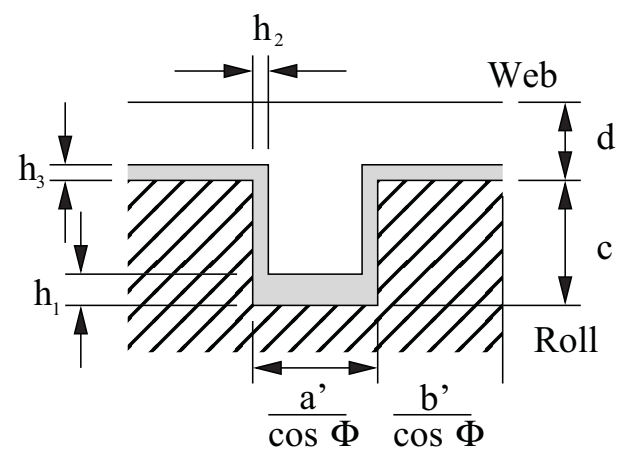

Fig. 3. Cross section view (looking into the bead from X marked on figure 1) of the upstream meniscus. 


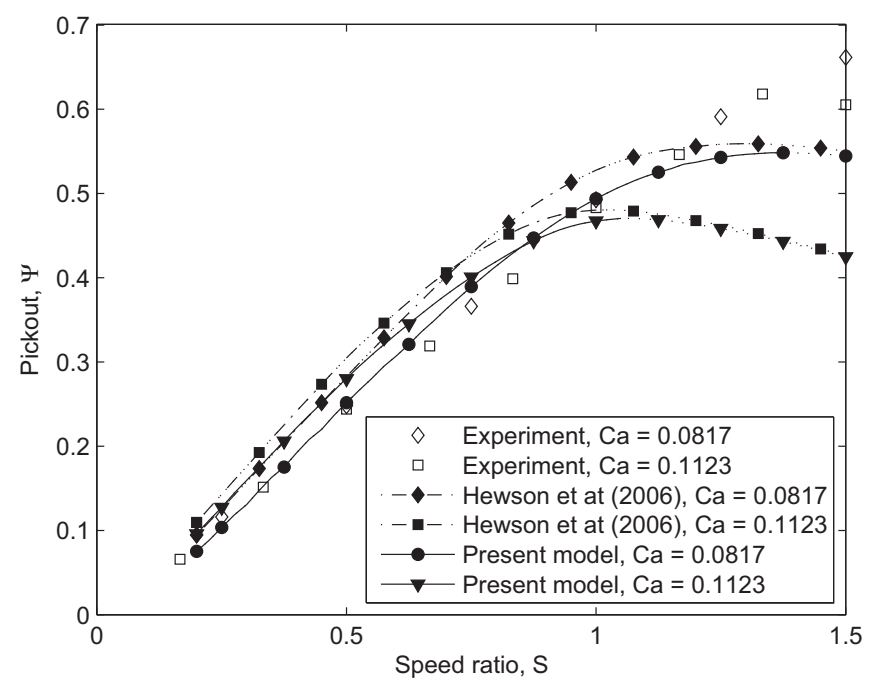

Fig. 4. Pickout as a function of speed ratio for roll R3: comparison between results obtained experimentally, one found using the theory of Hewson et al. (2006) and predictions from the present model; wrap angle $\beta=0^{\circ}$ static contact angle $\theta=18^{\circ}$. The dotted line indicates solutions for speed ratios greater than that for which maximum pickout occurs. 


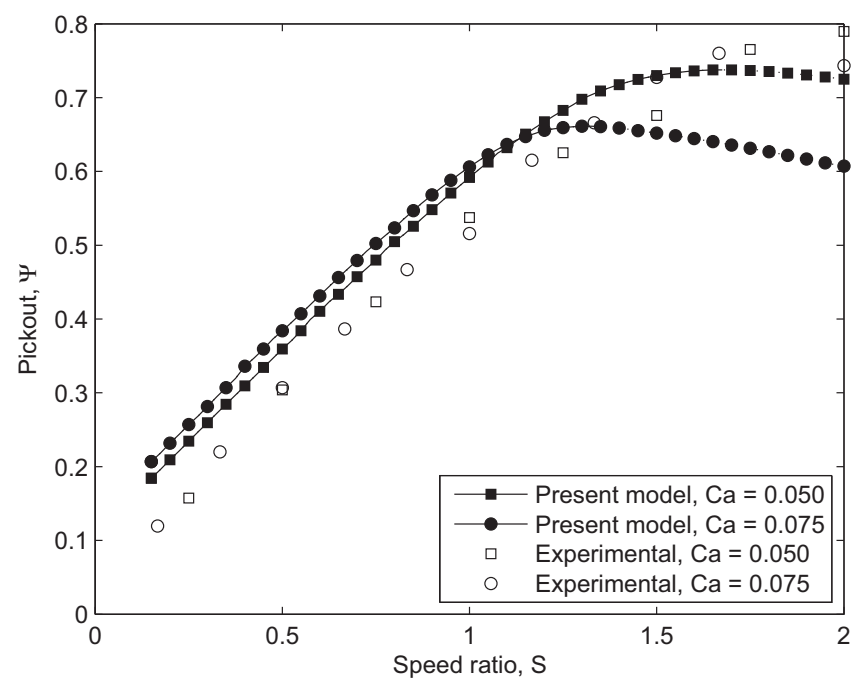

(a)

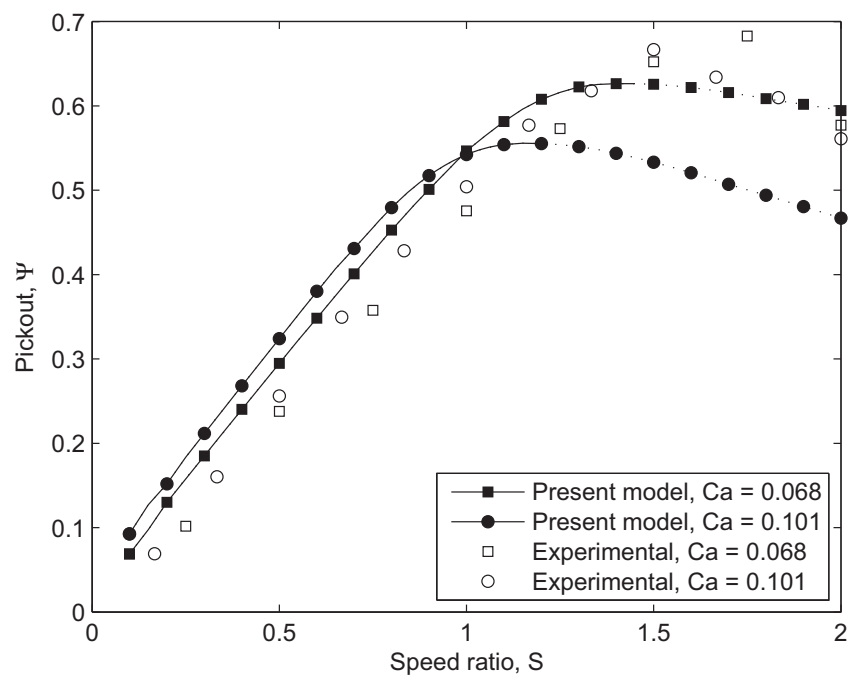

(b)

Fig. 5. Pickout predictions as a function of speed ratio for triangular grooves at zero pitch angle: (a) roll T2, $\beta=4.5^{\circ}$, (b) roll T3, $\beta=0^{\circ}$. The broken line indicates predictions of pickout for speed ratios greater than that for which the maximum pickout occurs. 


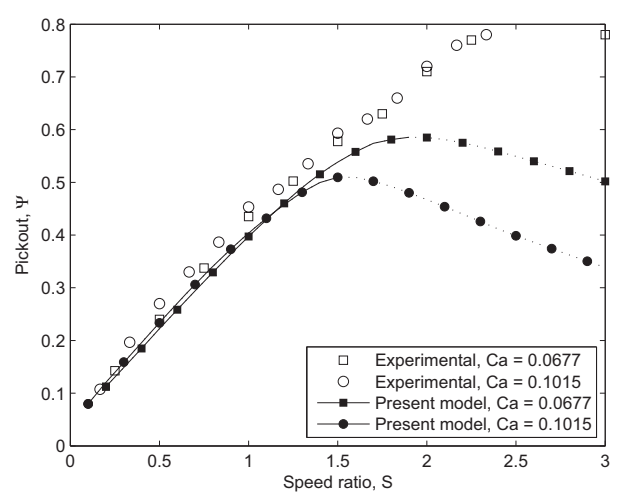

(a) Roll R4 $\left(\Phi=30^{\circ}\right), \beta=5^{\circ}$.

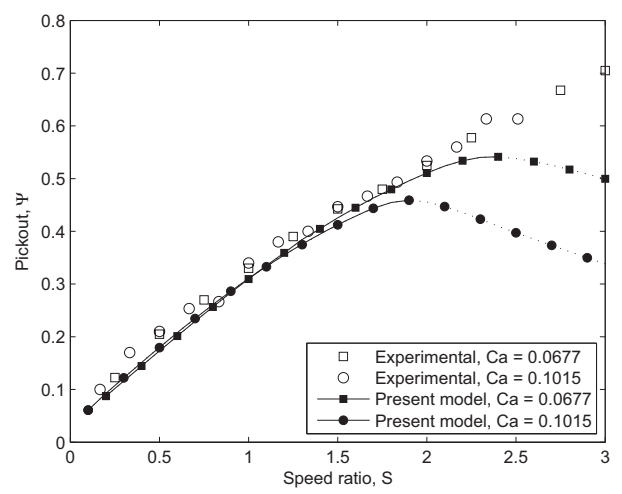

(c) Roll R5 $\left(\Phi=45^{\circ}\right), \beta=5^{\circ}$.

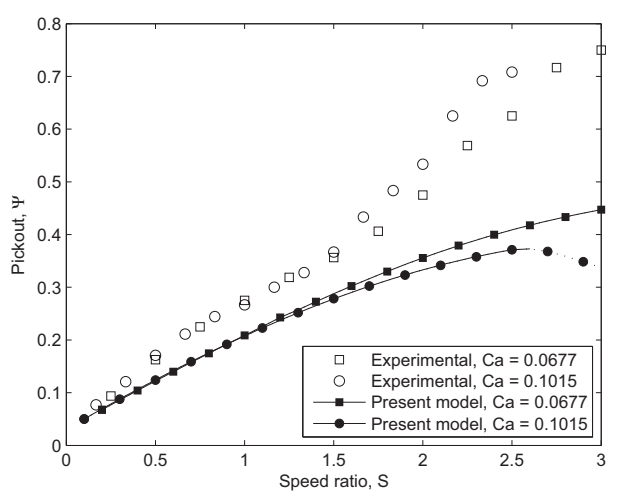

(e) Roll R6 $\left(\Phi=60^{\circ}\right), \beta=5^{\circ}$.

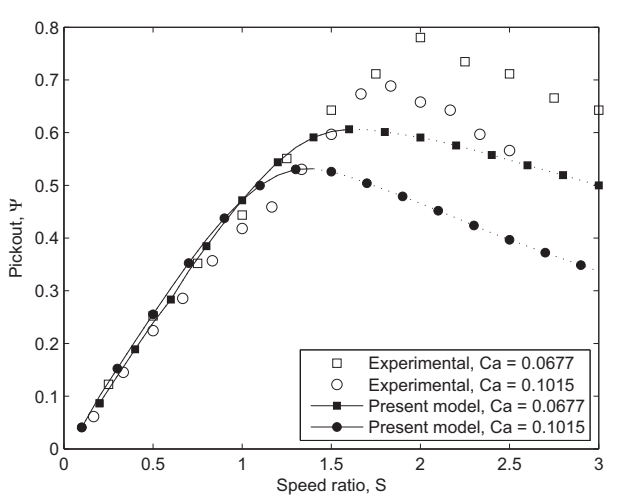

(b) Roll T4 $\left(\Phi=30^{\circ}\right), \beta=0^{\circ}$.

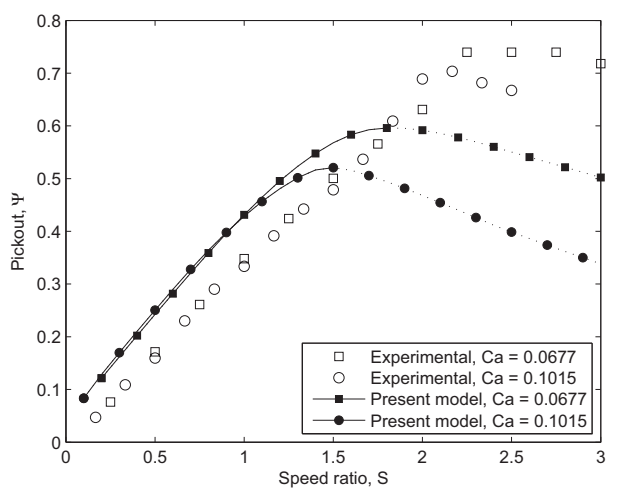

(d) Roll T5 $\left(\Phi=45^{\circ}\right), \beta=0^{\circ}$.

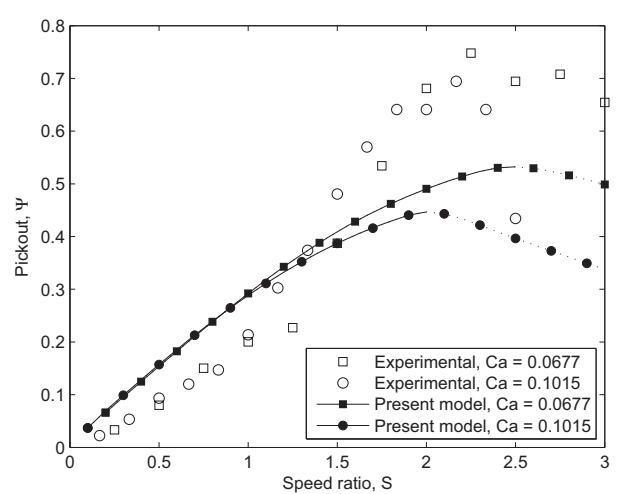

(f) Roll T6 $\left(\Phi=60^{\circ}\right), \beta=0^{\circ}$.

Fig. 6. Pickout predictions as a function of speed ratio for a range of rectangular (left hand column) and triangular (right hand column) grooves at non-zero angles of pitch. 


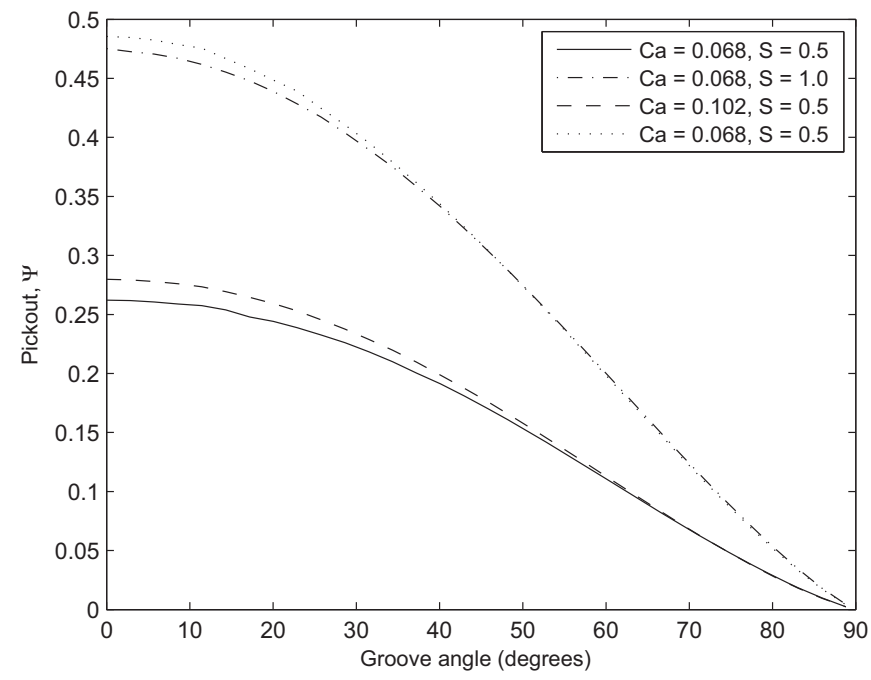

Fig. 7. Pickout predictions as a function of groove angle pitch, $\beta=5^{\circ}$. 


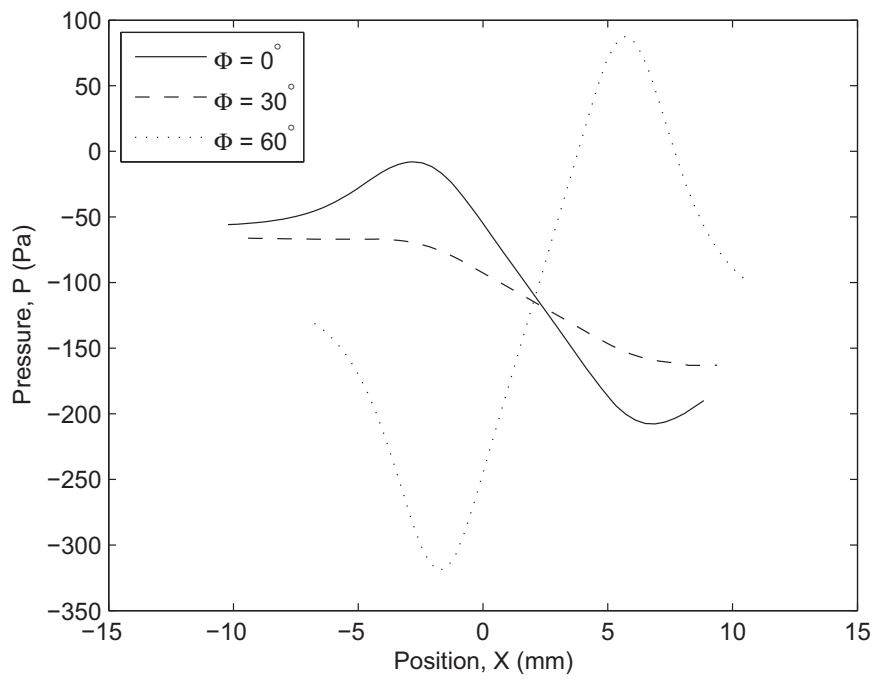

Fig. 8. Dimensional pressure distribution throughout the coating bead as a function of position for pitch angles of $0^{\circ}, 30^{\circ}$ and $60^{\circ}$; rectangular groove width $=0.5 \mathrm{~mm}$, depth $=$ $0.5 \mathrm{~mm}$ and land depth $=0.5 \mathrm{~mm} \mu=0.005 \mathrm{Pas}, \sigma=0.025 \mathrm{~N} / \mathrm{m}, \beta=5^{\circ}$ 


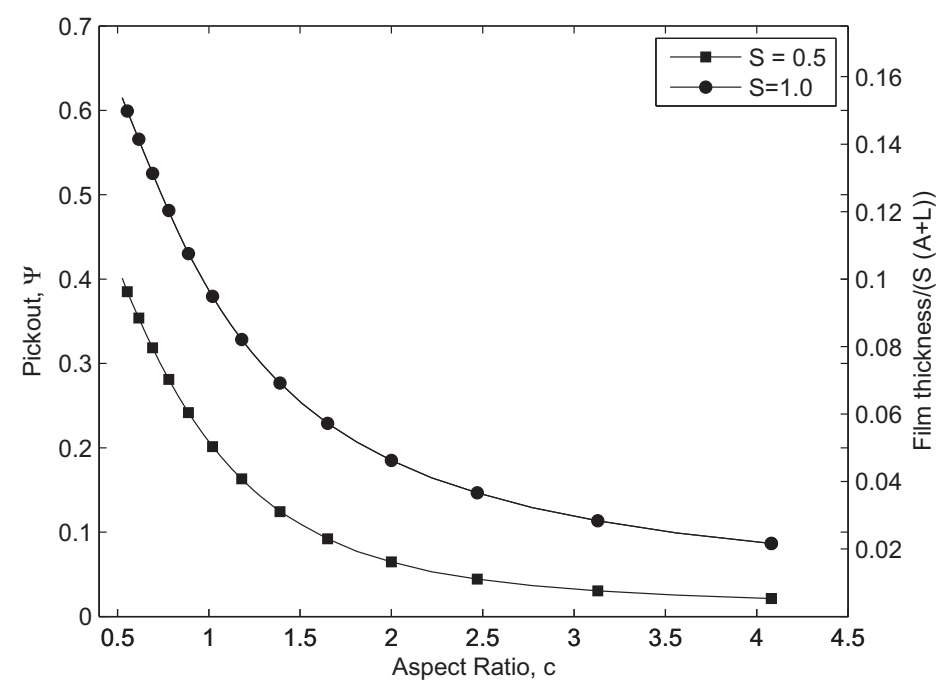

(a)

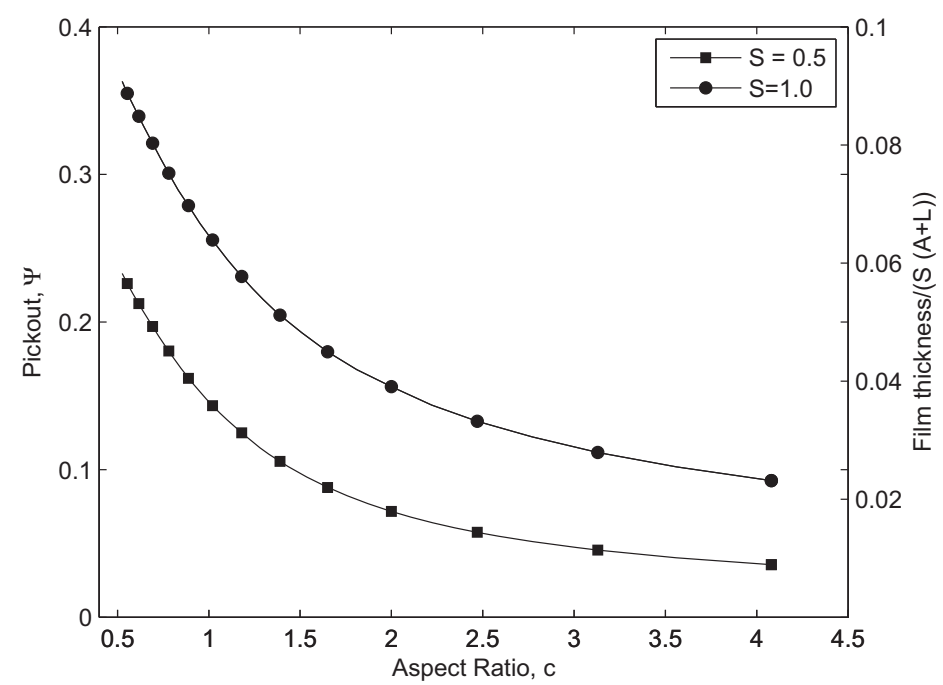

(b)

Fig. 9. Predictions of pickout as a function of groove aspect ratio for the case of $\beta=0^{\circ}$, Static Contact Angle, $\theta_{A}=45^{\circ}$, for groove pitch angles of (a) $\Phi=0^{\circ}$, (b) $\Phi=45^{\circ}$. 


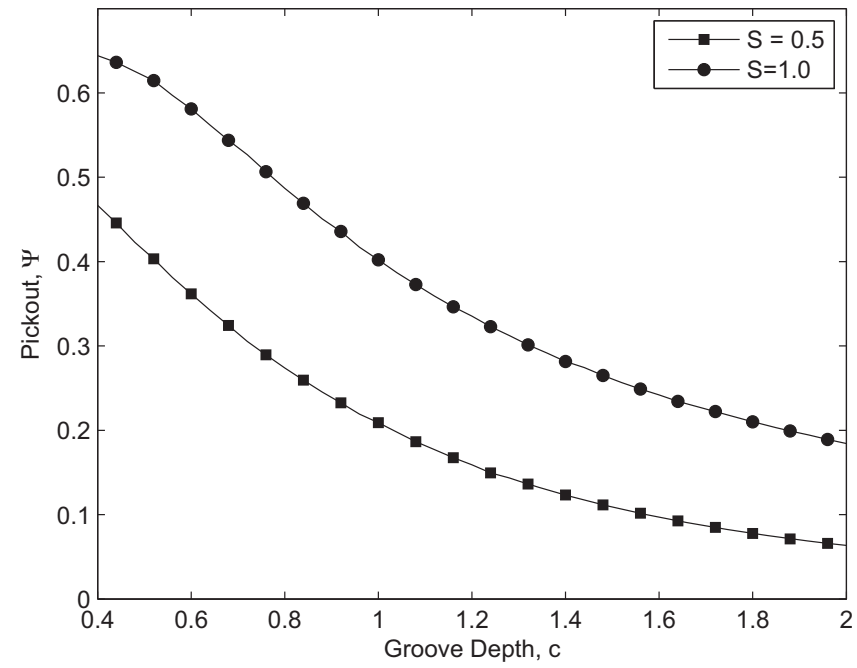

(a)

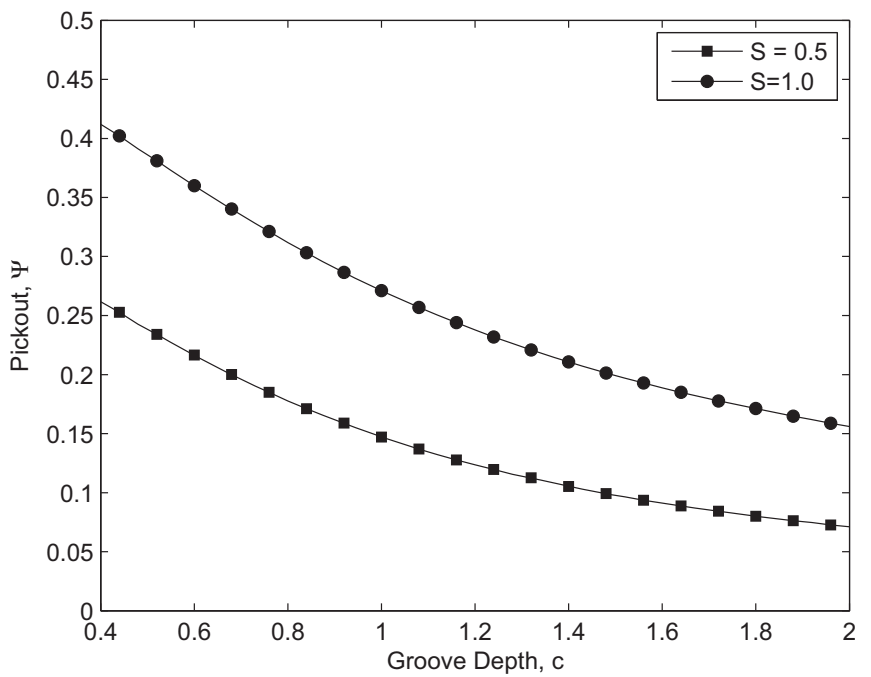

(b)

Fig. 10. Predictions of pickout as a function of groove depth for the case of rectangular grooves, Wrap Angle, $\beta=5^{\circ}$, static Contact Angle, $\theta_{A}=45^{\circ}$, for groove pitch angles of (a) $\Phi=0^{\circ}$, (b) $\Phi=45^{\circ}$ 


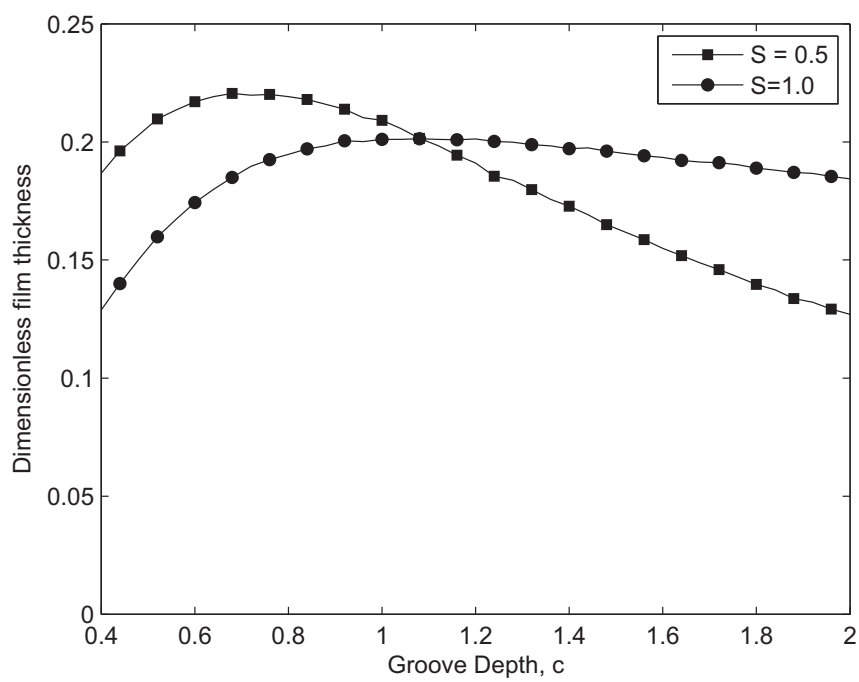

(a)

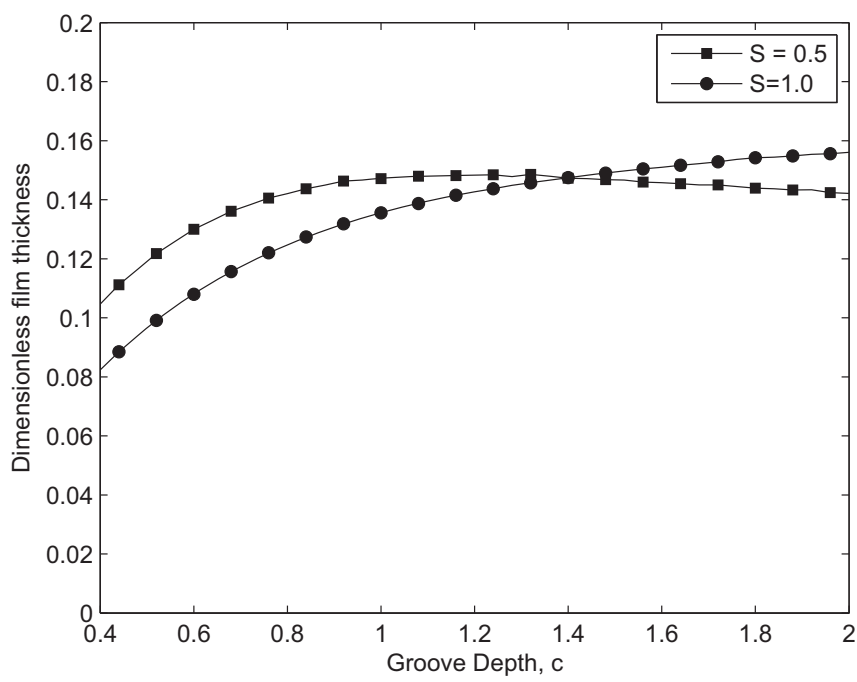

(b)

Fig. 11. Predictiosn of dimensionless film thickness as a function of groove depth: Rectangular Grooves, Roll radius, 50, Wrap Angle, $\beta=5^{\circ}$, Static Contact Angle, $\theta_{A}=45^{\circ}$, for groove pitch angles of (a) $\Phi=0^{\circ}$, (b) $\Phi=45^{\circ}$. 


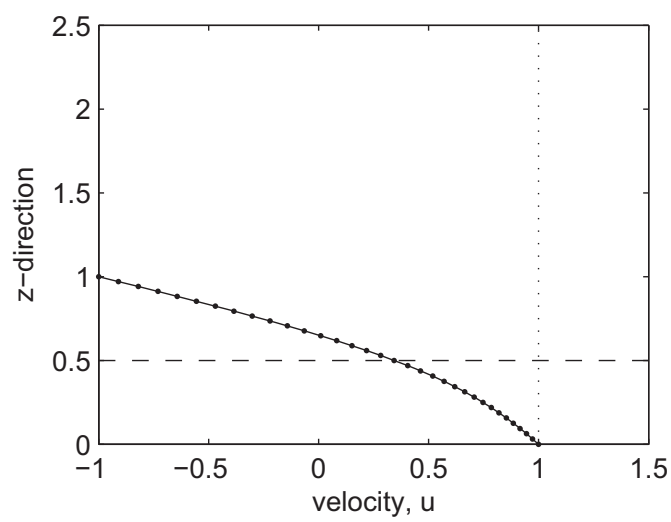

(a) $R=0.5$

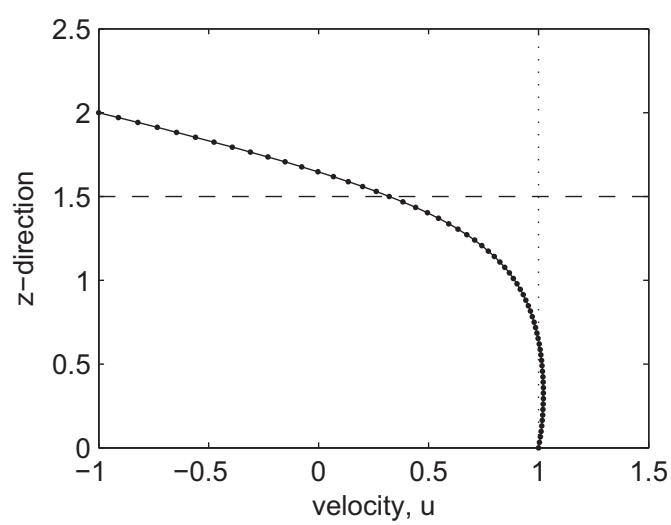

(c) $R=1.5$

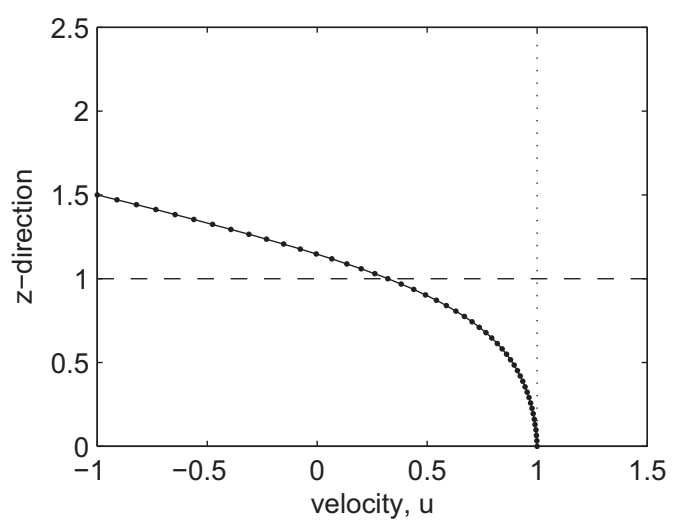

(b) $R=1.0$

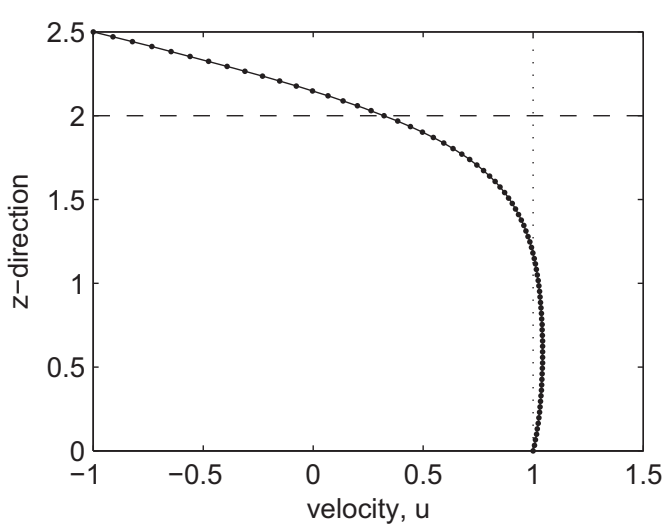

(d) $R=2.0$

Fig. 12. Velocity distribution taken down the centre of groove for different groove depths. Dotted lines $(\cdots)$ show roll velocity, dashed lines $(--)$ show the rolls land position. $(l=1$, $\left.b=1, d=0.5, S=-1, \frac{d p}{d x}=-0.5\right)$ 


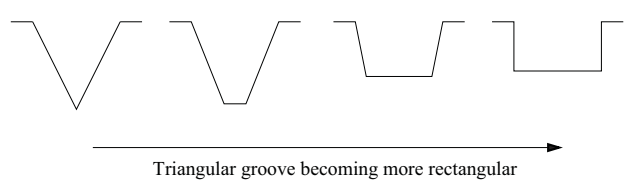

(a)
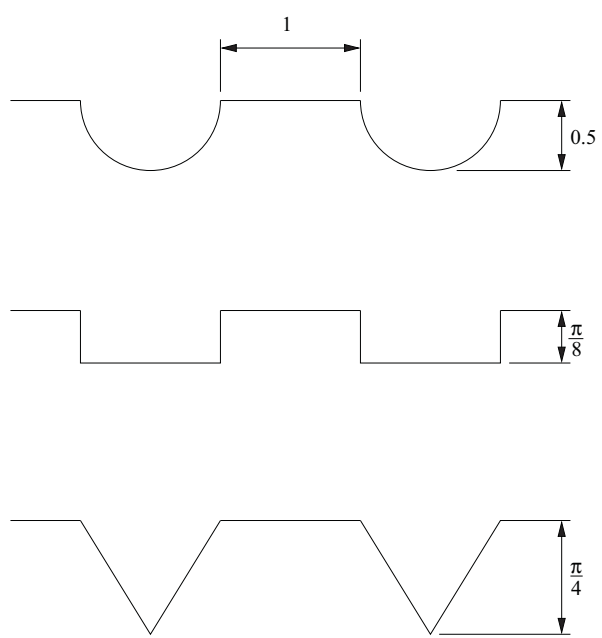

(b)

Fig. 13. Triangle to rectangle groove geometry transformation 


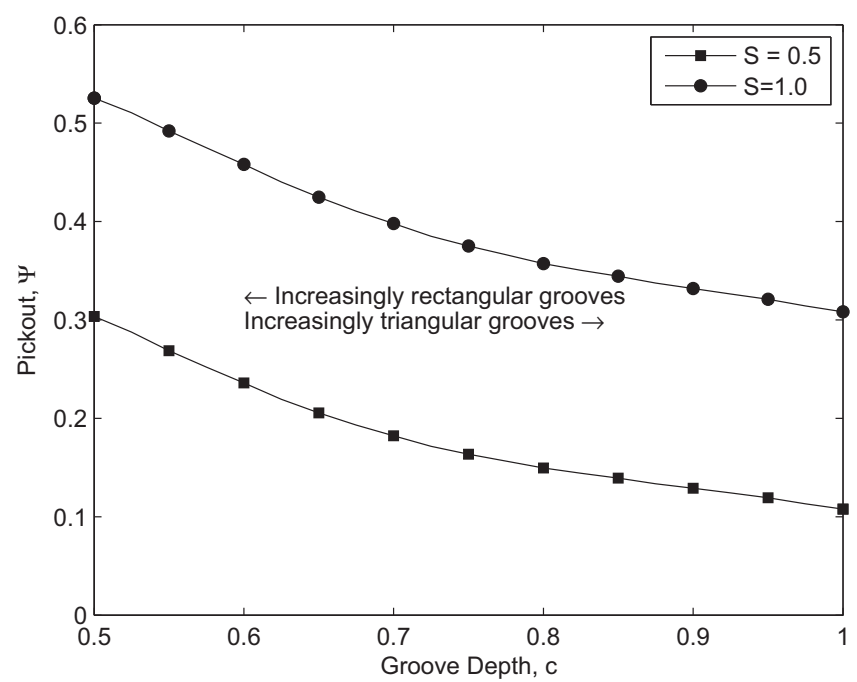

(a)

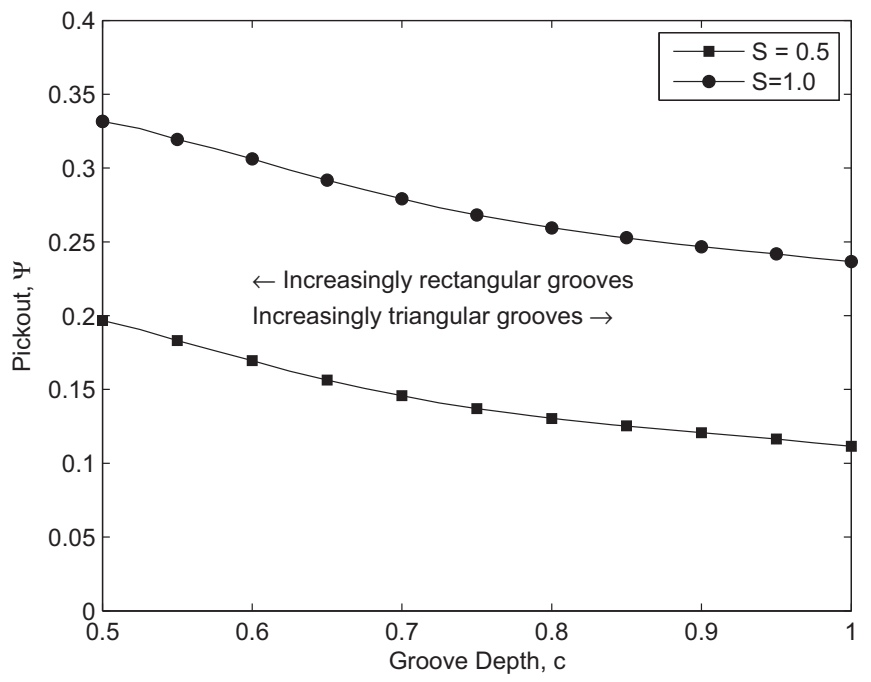

(b)

Fig. 14. Predicted pickout as a function of groove depth: Roll Radius, $r_{\text {roll }}=50$, Wrap Angle, $\beta=5^{\circ}$, Static Contact Angle, $\theta_{A}=60^{\circ}$, for groove pitch angles of (a) $\Phi=0^{\circ}$, (b) $\Phi=45^{\circ}$. 


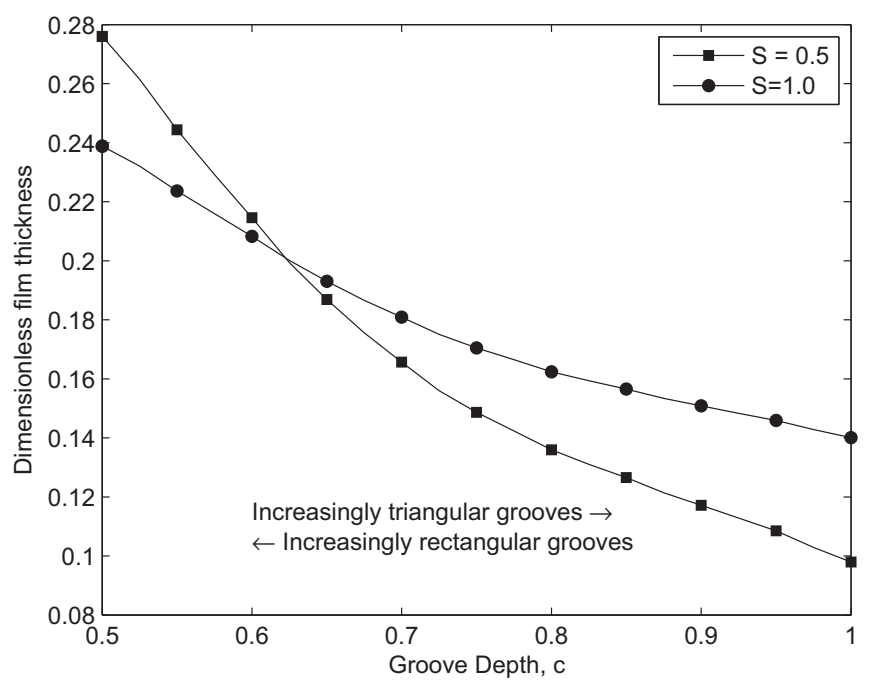

(a)

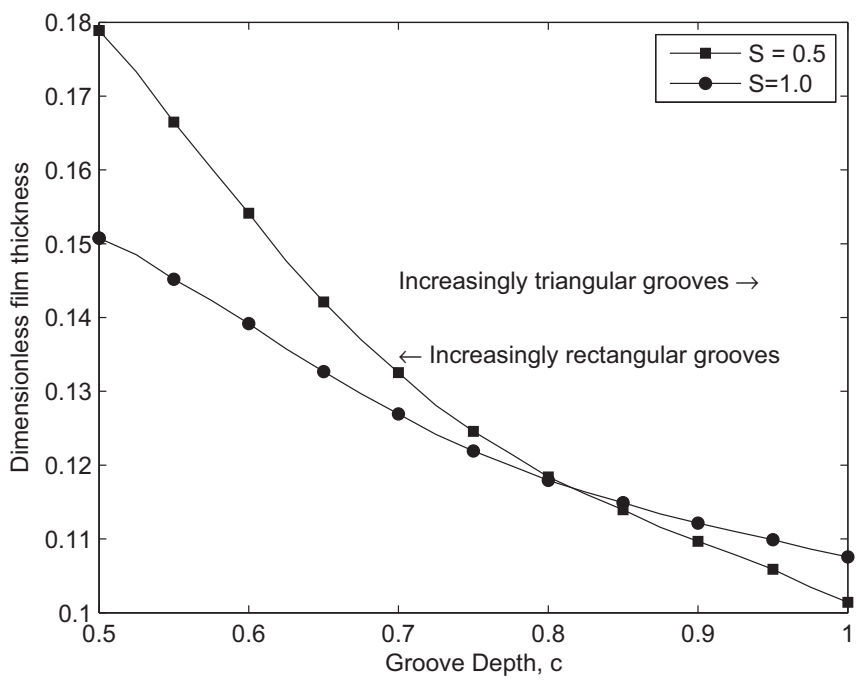

(b)

Fig. 15. Predicted dimensionless film thickness as a function of groove depth: Roll Radius, $r_{\text {roll }}=50$, Wrap Angle, $\beta=5^{\circ}$, Static Contact Angle, $\theta_{A}=60^{\circ}$ for groove pitch angles of (a) $\Phi=0^{\circ}$, (b) $\Phi=45^{\circ}$. 


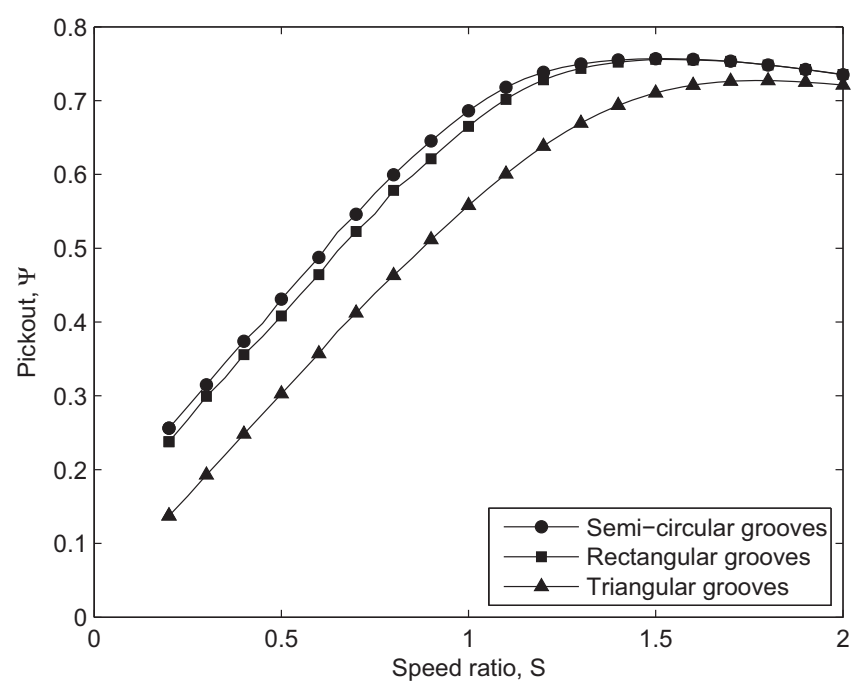

(a)

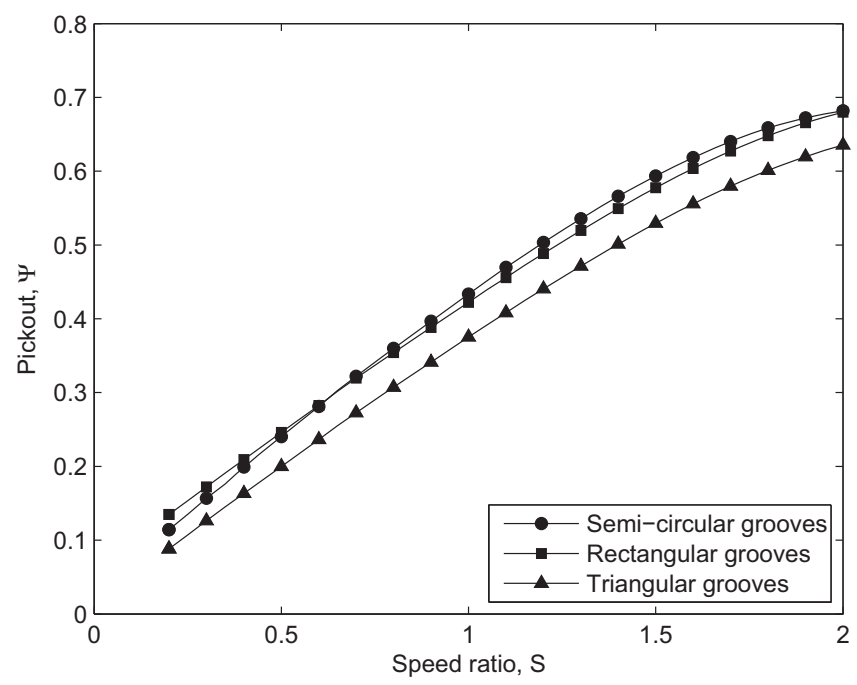

(b)

Fig. 16. Predicted pickout for equivalent triangular, rectangular and semi-circular groove geometries, as a function of speed ratio: Roll Radius, $r_{\text {roll }}=50$, Wrap Angle, $\beta=2.5^{\circ}$, Static Contact Angle, $\theta_{A}=45^{\circ}$, for groove pitch angles of (a) $\Phi=0^{\circ}$, (b) $\Phi=45^{\circ}$. 


\begin{tabular}{|c|c|c|c|c|c|}
\hline Roll & Groove Type & $\begin{array}{l}\text { Groove Width } \\
\qquad A(\mathrm{~mm})\end{array}$ & $\begin{array}{l}\text { Land Width } \\
\qquad B(\mathrm{~mm})\end{array}$ & $\begin{array}{l}\text { Groove Depth } \\
\qquad C(\mathrm{~mm})\end{array}$ & $\begin{array}{c}\text { Groove Pitch Angle } \\
\qquad \Phi\end{array}$ \\
\hline $\mathrm{R} 1$ & Rectangular & 0.47 & 0.53 & 0.18 & $0^{\circ}$ \\
\hline $\mathrm{R} 2$ & Rectangular & 0.47 & 0.54 & 0.35 & $0^{\circ}$ \\
\hline R3 & Rectangular & 0.47 & 0.53 & 0.30 & $0^{\circ}$ \\
\hline $\mathrm{R} 4$ & Rectangular & 0.50 & 0.50 & 0.40 & $30^{\circ}$ \\
\hline R5 & Rectangular & 0.50 & 0.50 & 0.40 & $45^{\circ}$ \\
\hline R6 & Rectangular & 0.60 & 0.40 & 0.40 & $60^{\circ}$ \\
\hline $\mathrm{T} 1$ & Triangular & 0.71 & 0.29 & 0.52 & $0^{\circ}$ \\
\hline $\mathrm{T} 2$ & Triangular & 0.82 & 0.18 & 0.60 & $0^{\circ}$ \\
\hline $\mathrm{T} 3$ & Triangular & 0.80 & 0.20 & 0.39 & $0^{\circ}$ \\
\hline $\mathrm{T} 4$ & Triangular & 0.25 & 0.33 & 0.30 & $30^{\circ}$ \\
\hline $\mathrm{T} 5$ & Triangular & 0.14 & 0.57 & 0.30 & $45^{\circ}$ \\
\hline T6 & Triangular & 0.29 & 0.79 & 0.30 & $60^{\circ}$ \\
\hline
\end{tabular}

Dimensions of the rectangular and triangular radially cut grooves. 
Table 2

\begin{tabular}{|c|c|}
\hline$A \times C$ & $0.5 \mathrm{~mm}^{2}$ \\
$R_{\text {roll }}$ & $50 \mathrm{~mm}$ \\
$\beta$ & $5^{\circ}$ \\
$A+B$ & $1 \mathrm{~mm}$ \\
$\theta_{A}$ & $74^{\circ}$ \\
$\Phi$ & 0 and $45^{\circ}$ \\
\hline
\end{tabular}

Roll dimensions for groove aspect ratio studies 